\title{
An Iterative Design with Variable Step Prototype Filter for Cosine Modulated Filter Bank
}

\author{
Samuel CHRIS PREMA ${ }^{1}$, Shubra Kankar DASGUPTA ${ }^{2}$ \\ ${ }^{1}$ Dept. of Avionics, Indian Institute of Space Science and Technology, Trivandrum, Kerala, India \\ ${ }^{2}$ Indian Institute of Space Science and Technology, Trivandrum, Kerala, India \\ chrisprema@iist.ac.in, ksd@iist.ac.in \\ Manuscript received March 31, 2015
}

\begin{abstract}
A systematic and self controlled prototype filter design approach for multichannel Cosine Modulated Near Perfect Reconstruction (NPR) filter bank is proposed in this paper. The primary goal is to design a prototype filter with enhanced performance i.e., minimum amplitude distortion and aliasing error. This algorithm approximates $3 d B$ cutoff frequency very close to $\pi / 2 M$. This is achieved by selecting suitable step size which is a function of transition width. If the selection of step size is too fine, the objective function oscillates. Whereas, if step size is coarse, $3 d B$ cutoff frequency will not be close to $\pi / 2 M$. This will degrade the overall performance of the prototype filter. Thus by choosing the step size as a function of transition width and varying the step size from coarser to finer level, the minimum amplitude distortion and aliasing error can be definitely achieved. The proposed filter is designed using two input parameters: number of subbands $M$ and attenuation $A$ and all other system parameters are derived from it to avoid heuristic inputs. Simulation results indicate better performance with reference to algorithms existing in literature. In addition, the design approach is systematic and self controlled.
\end{abstract}

\section{Keywords}

Filter bank, low pass prototype, subband, cosine modulation, NPR.

\section{Introduction}

Research in multichannel filter bank is gaining importance due to various applications in subband coding and transmultiplexer design for multimedia data transmissions. In general, the multichannel filter banks can be implemented using Cosine Modulated or Modified DFT filter banks [1], [2]. These filters can be built by complex modulation of a prototype filter [3]. An extensive research work is still continuing to find an optimal low pass prototype filter design approach. This plays a vital role in realization of filter bank structures. The filter bank design can be categorized into two types: Perfect Reconstruction (PR) and Near Perfect Reconstruction (NPR).

A large amount of work has been carried out to find an optimal prototype filter. Creusere and Mitra [4] have designed a prototype filter by optimizing the passband frequency with fixed filter length and relative error weighting. Lin and Vaidyanathan [5] have used the Kaiser Window approach for optimizing the cutoff frequency, and different window based technique with iterative algorithms are used in [6]. Also linear optimization technique is used in [7], [8] to optimize the $3 \mathrm{~dB}$ cutoff frequency with least square method. The samples in the transition band are optimized by frequency sampling in [10]. Some of the other approaches are second order cone programming [12], [13] and filter designs based on gradient information [14], [15]. But in most of the existing methods, a fixed filter order and an arbitrary step size are considered.

In this paper, a systematic and self controlled prototype filter design approach for Cosine Modulated filter bank is presented. The step size is varied from coarser to finer levels to achieve minimum amplitude distortion and aliasing error. The filter design requires two input parameters: number of subbands $M$ and subband attenuation $A$. All other required parameters are derived from these two input parameters. The NPR condition for the prototype filter is discussed in Sec. 2. The design procedure followed is given in Sec. 3. The simulations and results of the proposed method and comparison with the existing methods are discussed in Sec. 4. The contribution of the proposed scheme is concluded in Sec. 5 .

\section{NPR Condition for Prototype Filter}

For NPR filter banks, two conditions have to be satisfied by the prototype filter [2]. They are as follows:

(i) Prototype filter has to be band-limited as in (1)

$$
\left|H\left(e^{j \omega}\right)\right| \approx 0,|\omega|>\frac{\pi}{M} .
$$

ii) Frequency response of prototype filter has to be pairwise power complementary as in (2). 


$$
\left|H\left(e^{j \omega}\right)\right|^{2}+\left|H\left(e^{j\left(\frac{\pi}{M}-\omega\right)}\right)\right|^{2} \approx 1,0 \leq \omega \leq \frac{\pi}{M} .
$$

In general, the reconstructed signal differs from the input due to three reasons i) Aliasing, ii) Amplitude Distortion and iii) Phase Distortion. By choosing a linear phase FIR Type I filter, of length $N+1$ (where $N$ is even) the phase distortion can be eliminated completely. The amplitude distortion is reduced, when the band limiting condition stated in (1) is satisfied, and if the filters are pairwise power complementary as in (2), the aliasing error can also be reduced. The performance is evaluated by implementing an M-channel Cosine Modulated filter bank. The analysis and synthesis filters $h_{k}(n)$ and $f_{k}(n)$ are the Cosine Modulated versions of the prototype filter $h(n)$ and are given by (3) and (4) for $0 \leq n \leq N-1$ and $0 \leq k \leq M-1$.

$$
\begin{aligned}
& h_{k}(n)=2 h(n) \cos \left(\frac{\pi}{M}\left(k+\frac{1}{2}\right)\left(n-\frac{N-1}{2}\right)+(-1)^{k} \frac{\pi}{4}\right), \\
& f_{k}(n)=2 h(n) \cos \left(\frac{\pi}{M}\left(k+\frac{1}{2}\right)\left(n-\frac{N-1}{2}\right)-(-1)^{k} \frac{\pi}{4}\right) .
\end{aligned}
$$

The relation between the input signal $X(z)$ and the output signal $\hat{X}(z)$ is given in (5)

$$
\hat{X}\left(e^{j \omega}\right)=T_{o}\left(e^{j \omega}\right) X\left(e^{j \omega}\right)+\sum_{l=1}^{M-1} T_{l}\left(e^{j \omega}\right) X\left(e^{j \omega-2 \pi l / M}\right)
$$

where $\left|T_{o}\left(e^{j \omega}\right)\right|$ refers to amplitude distortion, and $T_{l}\left(e^{j \omega}\right)$ refers to the aliasing error.

\section{Design of Proposed Prototype Filter}

Literature survey reveals that researchers have worked out several efficient algorithms for design of low pass prototype filters in filter banks. However, most of the methods given in [4-9] start with an arbitrary fixed filter length and are optimized to get the NPR condition by varying the $3 \mathrm{~dB}$ cutoff frequency or pass band frequency with an arbitrary step size. Hence, no systematic procedure is followed for determining the step size. It is shown that the step size plays a significant role in achieving minimum amplitude distortion and aliasing error. The algorithm iteratively varies the step size from a coarser to finer level with a primary objective of arriving at minimum amplitude distortion and aliasing error.

This scheme uses step sizes as a function of the normalized transition width which is a function of the subbands $M$. The filter bank follows NPR condition of the prototype filter by controlling the magnitude response of the low pass prototype filter at $\pi / 2 M$ to be close to $1 / \sqrt{2}$.

$$
\left|H\left(e^{j \pi / 2 M}\right)\right|=1 / \sqrt{2} .
$$

The objective function given by (7) approximates the $3 \mathrm{~dB}$ cutoff frequency very close to the ideal filter

$$
\varphi=|| H\left(e^{j \pi / 2 M}\right)|-1 / \sqrt{2}| .
$$

Initially, the cutoff frequency is calculated using the relation $\omega_{\mathrm{c}}=\pi / 2 M$. The pass band can be approximated as $\omega_{\mathrm{p}} \approx \omega_{\mathrm{c}}=\pi / 2 M$ and stopband $\omega_{\mathrm{s}}=\pi / M$. Thus the approximate transition width $\left(\omega_{\mathrm{s}}-\omega_{\mathrm{p}}\right)$ is achieved as in (8) from the number of subbands $M$

$$
\Delta \omega=\pi / 2 M .
$$

The filter length $N$ can be obtained from the given attenuation $A$ in $\mathrm{dB}$ and number of subbands $M$ using (9)

$$
N \approx \frac{(A-7.95)}{14.36 \Delta \omega / 2 \pi} .
$$

In case of fixed length $N$, the transition width can be calculated using (10) from Kaiser Window Approach [5]:

$$
\Delta \omega=\frac{A-7.95}{2.285 N} .
$$

The step size is chosen as a fraction of the transition width $(\Delta \omega)$ in the proposed iterative prototype filter design algorithm.

\subsection{Design Procedure}

The systematic procedure followed for design of prototype filter is summarized below:

Given user input: Number of subbands $M$, attenuation $A$ in $\mathrm{dB}$ and Window function $W(n)$.

If filter length is given, the transition width can be obtained using (10) else, filter length can be determined using (9). [Note: Any Window function: Kaiser, Blackmann, Hamming may be chosen. Here, analysis is done with Kaiser Window.]

Step 1: Calculate:

(i) The $3 \mathrm{~dB}$ cut-off frequency: $\omega_{\mathrm{c}}=\pi / 2 M$

(ii) Transition width using (8).

Let step size, $\delta=f(\Delta \omega)$ [A fraction of the transition width $\Delta \omega$.

(iii) Initialize: error threshold;

Step 2 (a): Step size $\delta_{j}=\Delta \omega / l_{j}$ where $l_{j}=k_{1}+(j-1) k$; $j=1,2,3, \ldots, m ; k_{1}$ is the initial value and $k$ step increment.

[Note: Iteration must start with coarser step size and move to finer step size which implies $l_{j+1}>l_{j}$ and $\delta_{j+1}<\delta_{j}$.]

Step 2 (b): Iteration count for $\omega_{\mathrm{c}}$ updation : $i=1$ : count;

Step 3: Calculate: $h(n)$ - the lowpass prototype filter of length $N+1$, where $W(n)$ is the window function:

$$
h(n)=\sin (n-N / 2) \omega_{c} /(\pi(n-N / 2)) W(n) .
$$

Step 4: Calculate the objective function i.e., magnitude response at $3 \mathrm{~dB}$ 


$$
\varphi=\left|H\left(\frac{\pi}{2 M}\right)\right|-\frac{1}{\sqrt{2}} \mid .
$$

Condition_a $: \varphi>$ error threshold

Condition_b $: \varphi \leq$ error threshold

Condition_a_l: $\varphi(i+1)^{\text {th }}$ iteration $\leq \varphi(i)^{\text {th }}$ iteration

Condition_a_2: $\varphi(i+1)^{\text {th }}$ iteration $>\varphi(i)^{\text {th }}$ iteration

Step 5: If Cond_a go to Step 5(a) else go to Step 6

Step 5 (a): If Cond_a_l then $\omega_{c}=\omega_{c}+\delta_{j} ; i=i+1$ goto Step 3, until $i \leq$ count, [If $i>$ count go to Step 2 (a)] else Cond $\_\_2$ go to Step 2(a) to update step size $\delta_{j}$.

Step 6: Cond_b is satisfied, check for transition point with amplitude distortion/aliasing error using (11) or (12).

If error for the $(j+1)^{\text {th }}$ step size $\leq$ error for the $(j)^{\text {th }}$ step size then go to Step 2(a) to update the step size, else go to Step 7.

Step 7: Terminate; $h(n)$ with step size for the $(j)^{\text {th }}$ iteration is the final prototype filter.

Check for transition point: The step size should be varied from a coarser to a finer level to get the transition point where the filter would give minimum amplitude distortion and aliasing error.

In the existing methods [4-9] fixed step size is used in iteration. Hence, there is no mechanism to find the minimum distortion unless the step size is chosen appropriately. Whereas in the proposed method, the step sizes are varied from coarser to finer level to systematically arrive at a minimum amplitude distortion and aliasing error. Hence, this algorithm becomes structured and does not require any heuristic input. The performance of the proposed prototype filter design approach is determined by evaluation of maximum peak ripple $R_{p}$ using (11), and the maximum aliasing error $\varepsilon_{a}$ using (12) as in [7] where

$$
R_{p}=\max \left(1-\left|T_{o}\left(e^{j \omega}\right)\right|\right)
$$

$R_{p}$ measures the worst amplitude distortion.

$$
\varepsilon_{a}=\max \left(T_{l}\left(e^{j \omega}\right)\right) \text { for } \in[0, \pi], 1 \leq l \leq M-1
$$

where $T_{l}\left(e^{j \omega}\right)=\frac{1}{M} \sum_{k=0}^{M-1} F_{k}\left(e^{j \omega}\right) H_{k}\left(e^{j \omega-\frac{2 \pi l}{M}}\right)$ and $T_{l}\left(e^{j \omega}\right)$ refers to the aliasing error.

\section{Design Examples and Simulation Results}

\subsection{Comparison of Filter Performance}

The proposed prototype filter was designed for varying number of subbands $M$ fixed filter length $N$ and a pre-

\begin{tabular}{|l|l|l|l|}
\hline Type of algorithm & $\begin{array}{l}\text { Filter } \\
\text { Length } \boldsymbol{N}\end{array}$ & $\begin{array}{l}\text { Amplitude } \\
\text { Distortion } \boldsymbol{R}_{\boldsymbol{p}}\end{array}$ & $\begin{array}{l}\text { Aliasing } \\
\text { Error } \boldsymbol{\varepsilon}_{\boldsymbol{a}}\end{array}$ \\
\hline Algorithm in [4] & 439 & $1.80 \times 10^{-3}$ & $1.96 \times 10^{-6}$ \\
\hline Algorithm in [5] & 467 & $2.42 \times 10^{-3}$ & $2.76 \times 10^{-7}$ \\
\hline Algorithm in [6] & 439 & $3.06 \times 10^{-3}$ & $1.85 \times 10^{-7}$ \\
\hline Algorithm in [7] & 448 & $1.50 \times 10^{-3}$ & $9.45 \times 10^{-7}$ \\
\hline Algorithm in [8] & 440 & $3.42 \times 10^{-3}$ & $2.60 \times 10^{-7}$ \\
\hline Algorithm in [9] & 512 & $1.01 \times 10^{-3}$ & $5.66 \times 10^{-7}$ \\
\hline Proposed method & $\mathbf{4 3 9}$ & $\mathbf{1 . 0 9 5 4 \times \mathbf { 1 0 } ^ { - 4 }}$ & $\mathbf{3 . 0 4 1 \times \mathbf { 1 0 } ^ { - 8 }}$ \\
\hline
\end{tabular}

Tab. 1. Comparison with existing methods [4-9] for subbands $\mathrm{M}=32$.

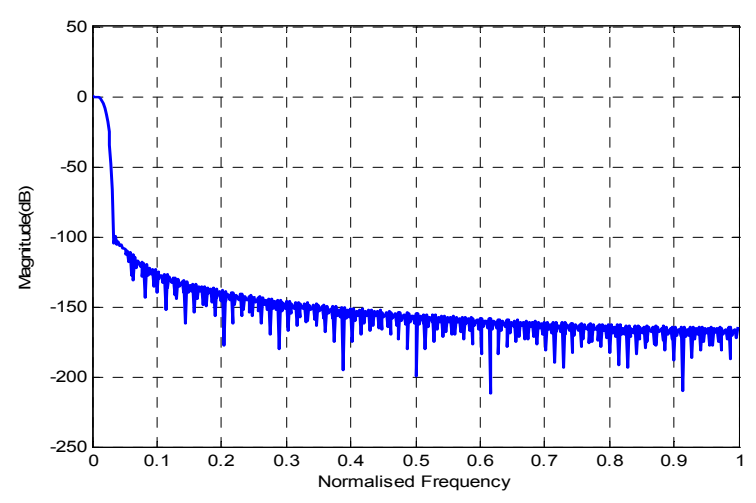

Fig. 1. The frequency response of prototype filter for $M=32$.

defined stopband attenuation of $100 \mathrm{~dB}$. The results of this method were compared with other existing methods in literature [4-9] for $M=32$, fixed filter length $N=439$. The results obtained are tabulated in Tab. 1. The frequency response of the prototype filter designed for $M=32$, is shown in Fig. 1. Table 2 shows the performance of the filter designed with attenuation $A=100 \mathrm{~dB}$ for different subbands $[M=8,16,32,64]$, with fixed filter length. Table 3 summarizes the performance of the prototype filter design for different subbands $[M=8,16,32,64]$ with the appropriately evaluated filter length using (9).

\subsection{Impact of Variable Step Size on Filter Performance}

Impact of variable step size on filter performance is explained by varying the step size from coarser to finer granularity. This is shown in Fig. 2 for filter length $N=439$ and subbands $M=32$ for variable step sizes $\left(4.55 \times 10^{-4}, 9.2 \times 10^{-4}, 1.84 \times 10^{-3}\right)$ with iterations 6,11 , 19. Step sizes are varied when the error conditions are not satisfied. Hence, the algorithm becomes computationally efficient and self controlled. It is apparent from Fig. 2 that the minimum amplitude distortion is at transition point. The impact of variable step sizes and the different transition points for variable filter length $N$ and subbands $M$ are shown in Fig. 3, where the performance is shown in Fig. 3(a) for peak amplitude distortion and in Fig. 3(b) for minimum aliasing error. Hence, one may conclude that proper selection of step size is an essential to arrive at a minimum amplitude distortion and aliasing error for different values of subbands $M$. 


\begin{tabular}{|l|l|l|}
\hline Subbands $\boldsymbol{M}$ & $\begin{array}{l}\text { Amplitude } \\
\text { Distortion } \boldsymbol{R}_{\boldsymbol{p}}\end{array}$ & $\begin{array}{l}\text { Aliasing } \\
\text { Error } \boldsymbol{\varepsilon}_{\boldsymbol{a}}\end{array}$ \\
\hline 64 & $2.4429 \times 10^{-4}$ & $8.994 \times 10^{-8}$ \\
\hline 32 & $1.0954 \times 10^{-4}$ & $3.041 \times 10^{-8}$ \\
\hline 16 & $1.1051 \times 10^{-4}$ & $2.164 \times 10^{-7}$ \\
\hline 8 & $1.0126 \times 10^{-4}$ & $2.128 \times 10^{-7}$ \\
\hline
\end{tabular}

Tab. 2. Performance of the proposed method with different number of subbands $M$ for a filter length of 439 .

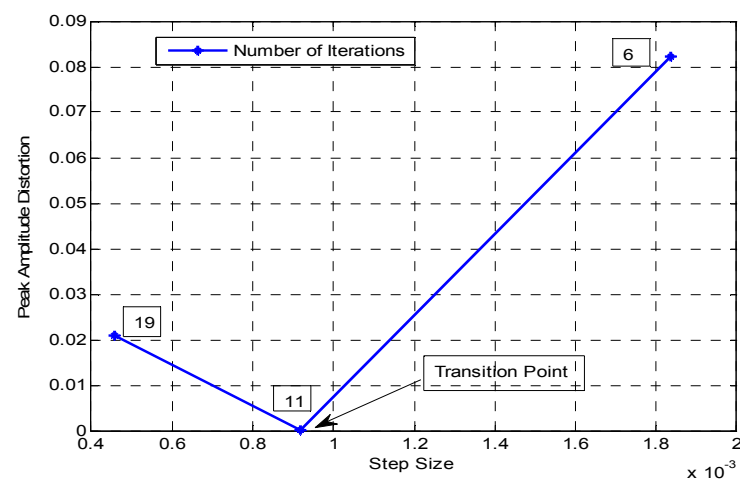

Fig. 2. Effect of step size on Peak Amplitude Distortion of the proposed method for subbands $M=32$ and filter length $N=439$.

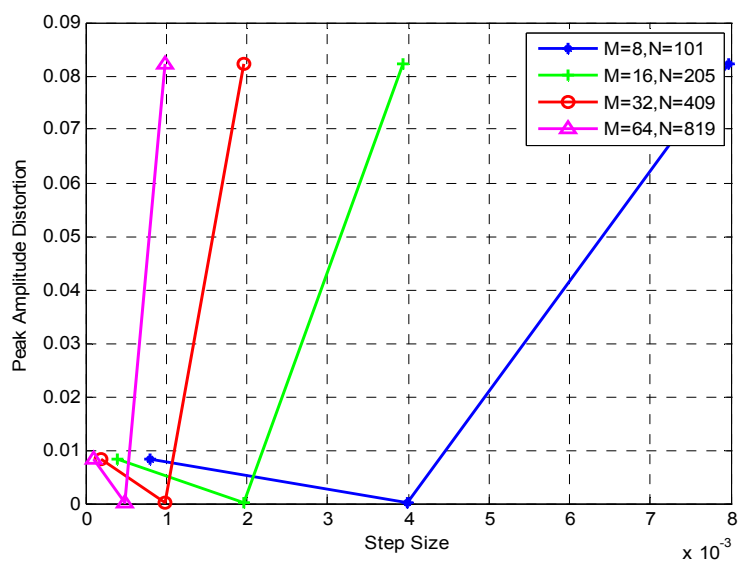

(a)

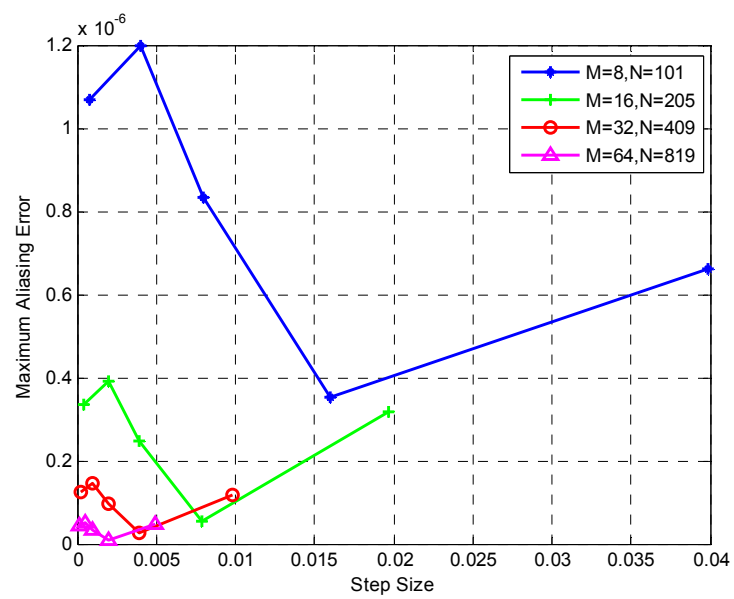

(b)

Fig. 3. The significance of transition point variation for the proposed method with different filter length $N$ and subbands M. (a) Amplitude Distortion, (b) Maximum Aliasing Error.

\begin{tabular}{|l|l|l|l|}
\hline $\begin{array}{l}\text { Subbands } \\
\boldsymbol{M}\end{array}$ & $\begin{array}{l}\text { Filter } \\
\text { Length } \boldsymbol{N}\end{array}$ & $\begin{array}{l}\text { Amplitude } \\
\text { Distortion } \boldsymbol{R}_{\boldsymbol{p}}\end{array}$ & $\begin{array}{l}\text { Aliasing } \\
\text { Error } \boldsymbol{\varepsilon}_{\boldsymbol{a}}\end{array}$ \\
\hline 64 & 819 & $1.2106 \times 10^{-4}$ & $5.11 \times 10^{-8}$ \\
\hline 32 & 409 & $1.2106 \times 10^{-4}$ & $1.45 \times 10^{-7}$ \\
\hline 16 & 205 & $1.2108 \times 10^{-4}$ & $3.92 \times 10^{-7}$ \\
\hline 8 & 101 & $1.1747 \times 10^{-4}$ & $1.20 \times 10^{-6}$ \\
\hline
\end{tabular}

Tab. 3. Performance of the proposed method with different subbands $M$ and filter length $N$ evaluated using Kaiser Window.

\section{Conclusion}

A systematic and self controlled algorithm to design a low pass linear phase FIR prototype filter for NPR Cosine Modulated filter bank is presented in this paper. In this scheme, the prototype filter cut-off frequency is approximated to the $3 \mathrm{~dB}$ response iteratively. The step size is varied as a function of transition width instead of an arbitrary step size. By varying the step size from coarser to finer levels, the minimum amplitude distortion and aliasing error can be definitely achieved. The prototype filter is designed with two user inputs - number of subbands $M$ and attenuation $A$. The performance of the proposed method was compared with other methods existing in the literature through exhaustive simulation. The results indicate that the method presented in this paper is better in terms of amplitude distortion and aliasing error. This approach is simple and systematic compared to other widely used techniques. In addition, this algorithm is structured and does not call for any heuristic input.

\section{References}

[1] VAIDYANATHAN, P. P. Multirate Systems and Filter Banks. Englewood Cliffs, NJ: Prentice-Hall, 1993. ISBN 10/ISBN 13: 01360571879780136057185

[2] KARP, T., FLIEGE, N. J. Modified DFT filter banks with perfect reconstruction. IEEE Transactions on Circuits and Systems, 1999, vol. 46, no. 11, p. 1404-1414. DOI: 10.1109/82.803480

[3] KOILPILLAI, R. D., VAIDYANATHAN, P. P. Cosine-modulated FIR filter banks satisfying perfect reconstruction. IEEE Transactions on Signal Processing, 1992, vol. 40, no. 4, p. 770-783. DOI: 10.1109/78.127951

[4] CREUSERE, C. D., MITRA, S. K. A simple method for designing high-quality prototype filters for M-band pseudo QMF banks. IEEE Transactions on Signal Processing, 1995, vol. 43, no. 4, p. 1005-1007. DOI: 10.1109/78.376856

[5] LIN, Y. P., VAIDYANATHAN, P. P. A Kaiser window approach for the design of prototype filters of cosine modulated filter banks. IEEE Signal Processing Letters, 1998, vol. 5, no. 6, p. 132-134. DOI: $10.1109 / 97.681427$

[6] CRUZ ROLDAN, F., AMO-LOPEZ, P., MALDONADO-BASCON, S., LAWSON, S. S. An efficient and simple method for designing prototype filters for cosine modulated pseudo-QMF banks. IEEE Signal Processing Letters, 2002, vol. 9, no. 1, p. 29-31. DOI: $10.1109 / 97.988722$

[7] KUMAR, A., SINGH, G. K., ANAND, R. S. A simple design method for the cosine modulated filter bank using weighted least 
square technique. Journal of the Franklin Institute, 2011, vol. 348, p. 606-621. DOI: 10.1016/j.franklin.2011.01.006

[8] BERGEN, S. W. A., ANTONIOU, A. An efficient closed form design method for cosine modulated filter banks using window functions. Signal Processing, 2007, vol. 87, no. 5, p. 811-823. DOI: $10.1016 /$ j.sigpro.2006.08.003

[9] BERGEN, S. W. A. A design for cosine modulated filter banks using weighted constrained least squares filters. Digital Signal Processing, 2008, vol. $18, \quad$ no. $3, \quad$ p. 282-290. DOI: 10.1016/j.dsp.2007.04.010

[10] DATAR, A., JAIN, A., SHARMA, P. C. Design of Kaiser window based optimized prototype modulated filter banks. Signal Processing, 2010, vol. 90, no. 5, p. 1742-1749. DOI: 10.1016/j.sigpro.2009.11.011

[11] CRUZ-ROLDAN, F., SANTAMARIA, I., BRAVO, A. M. Frequency sampling design of prototype filters for nearly perfect reconstruction cosine modulated filter banks. IEEE Signal Processing Letters, 2004, vol. 11, no. 3, p. 397-400. DOI: 10.1109/LSP.2003.821663

[12] DOBLINGER, G. A fast design method for perfect-reconstruction uniform cosine modulated filter banks. IEEE Transactions on Signal Processing, 2012, vol. 60, no. 12, p. 6693-6697. DOI: 10.1109/TSP.2012.2217139

[13] LU, W.S., SARAMÄKI, T., BREGOVIC, R. Design of practically perfect-reconstruction cosine-modulated filter banks: A secondorder cone programming approach. IEEE Transactions on Circuits and Systems I, 2004, vol. 51, no. 3, p. 552-563. DOI: 10.1109/TCSI.2003.822555
[14] ZHANG, Z.J. Efficient design of cosine modulated filter banks based on gradient information. IEEE Signal Processing Letters, 2007, vol. 14, no. 12, p. 940-943. DOI: 10.1109/LSP.2007.906624

[15] ZHANG, Z.J., SHUI, P.L., SU, T. Efficient design of high complexity cosine modulated filter banks using $2 \mathrm{Mth}$ band conditions. IEEE Transactions on Signal Processing, 2008, vol. 56, no. 11, p. 5414-5426. DOI: 10.1109/TSP.2008.929672

\section{About the Authors ...}

Samuel CHRIS PREMA was born in 1979. She received her Bachelor's degree in Electronics and Communication and Master's degree in Applied Electronics. Her research interests include communication and multirate filter banks.

Shubra Kankar DASGUPTA was born in 1951. He received his Bachelor's Degree in Electronics and Telecommunications and his Master's degree in Computer Science from Jadavpur University. He received his Ph.D. in Electrical Engineering from the Indian Institute of Technology, Bombay. His research interests include image processing and satellite communications. He is currently the Director of the Indian Institute of Space Science and Technology, Thiruvananthapuram. He has also served as the Deputy Director, Space Applications Centre, Ahmadabad and the Director of the Development and Educational Communication Unit of Indian Space Research Organization. 